\title{
(Un-)Sicherheitsproduzent und Differenzmaschine? Erweiterte Perspektiven auf die Geschichte der bundesdeutschen Polizei nach $1945^{1}$
}

Marcel Schmeer

\section{Abstract}

There is hardly another organisation that lends itself so impressively to tracing the relationship between the production of (in-)security and difference than the police as a "differentiating apparatus". In going about their daily business of maintaining public security and order, the representatives of the state monopoly over the means of violence continuously (re-)construct, apply and reinforce criteria of difference, which has lately rendered the police the target of profound criticism - expressed, for instance, in the form of the global Black Lives Matter protests against police violence and practices of racial profiling. The logics underlying these differentiation practices among the Federal Republic's police have undergone historical change, owing both to the push of external factors and internal reform efforts. The police force itself has also become more diversified over time, which has entailed new challenges of its own. Starting from these initial considerations, this contribution adopts a broader perspective of the history of the Federal Republic's police force as a reflection of the history of German society. With an eye to the three categories of difference of gender, race/ethnicity and sexuality, the article traces the multifaceted and contradictory points of intersection between difference and security by examining the historical example of West Berlin's police and its conflictual relationship with the city's population in a social setting marked by tension between mainstream society and its minorities. It inquires into the gender images and relations within and outside the police, the relationship between the police and "foreigners" in a rapidly diversifying West Berlin in the 1970s and the relationship between the police and homosexuals in the metropolis on the Spree river. The ultimate objective of this contribution is to sensitise us toward the manifold relationships of difference and the associated conceptions of security in the everyday practice of policing as well as within the organisation itself.

1 Für konstruktive Kritik, weiterführende Kommentare und Hinweise sowie aufmerksames Korrekturlesen danke ich sehr herzlich meinen Kolleg*innen Marcus Böick, Marc Frey, Julius Heise, Eva Herschinger, Christopher Kirchberg und Paula Lange sowie den geduldigen Herausgeberinnen dieses Sammelbandes Anja Krause und Sigrid Ruby. 


\section{Einleitung}

Befeuert von Protesten und Debatten in den USA ist auch die deutsche Polizei in letzter Zeit unter anderem wegen des Verdachts des racial profiling in die öffentliche Kritik geraten, der diskriminierenden Ungleichbehandlung von Personen durch Polizeibeamt*innen aufgrund äußerer Merkmale, etwa im Zuge von Kontrollen ohne konkreten Anlass (vgl. Hunold/ Wagner 2020, S. 29f.; Zeit Online 2020). Im Kern kreiste die Diskussion um die Frage, ob struktureller Rassismus auch in der Bundesrepublik tief in die Polizei und ihre Organisationskultur eingeschrieben sei - eine Frage, der sich nur mit Blick auf die historische Entwicklung dieser umstrittenen Institution der Inneren Sicherheit auf den Grund gehen lässt.

Dieser dynamische Gegenwartsimpuls verweist auf die konzeptionelle Stoßrichtung dieses Sammelbands, der von der Grundannahme ausgeht, dass (staatliche) Sicherheitskonzeptionen sich immer auch sozio-kultureller Differenzlogiken bedienen, diese verstärken und/oder (re-)kalibrieren. Auf diese Weise trügen sie zur Stabilisierung hegemonialer Konzepte der Mehrheitsgesellschaft bei (vgl. die Einleitung von Sigrid Ruby). Dies gilt in hohem Maße für die mit der (inneren) Sicherheit eines Staates betrauten Behörden und insbesondere die Polizei, deren uniformiertes Personal den Bürger*innen als sichtbare Repräsentanz des staatlichen Gewaltmonopols unmittelbar gegenübertritt. Bereits das öffentliche Image dieser „Institution öffentlicher Gewalt“ (Brusten/Feest/Lautmann 1975) lässt sich als widersprüchlich, geradezu janusköpfig beschreiben und changiert zwischen einer Wahrnehmung als notwendiger Sicherheitsproduzent und potenzielle Gefahrenquelle. Polizeikritische Sozialwissenschaftler*innen haben in den letzten Jahren mit Nachdruck betont, dass sich die Praxis der Aufrechterhaltung öffentlicher Sicherheit und Ordnung im Alltag immer in einem Spannungsverhältnis zwischen Hegemonie und Marginalisierung, Schutz und Schikane bewege. Der Mehrheit der Gesellschaft gilt die Polizei nach wie vor als „Freund und Helfer“ oder spielt für sie im Alltag, jenseits von Bagatellen, kaum eine Rolle. Diese Wahrnehmung bewirkt, dass sich Angehörige der Majoritätsgruppe häufiger mit einer polizeilichen Perspektive identifizieren oder damit zumindest sympathisieren können. Für Minderheiten und marginalisierte Gruppen einer Gesellschaft ist die Polizei im alltäglichen Leben oft präsenter und wird häufig als bedrohliche Willkürinstanz wahrgenommen (vgl. Loick 2018, S. 20-23).

Insbesondere diese zweite Wahrnehmungsfacette ist eng mit der Frage nach Differenzierungsprozessen und Versicherheitlichungsdynamiken verwoben (vgl. Buzan et al. 1997; Conze 2005; Hansen 2000). Die Polizei tritt im öffentlichen Diskurs wie in ihrer Alltagspraxis als unablässige Diffe- 
renzmaschine auf, die stets die gegenwärtigen (sicherheits-)politischen Grenzen von Mainstream und Devianz zu überwachen und deren Überschreitung (gewaltsam) zu sanktionieren hat. In dieser Funktion obliegt Polizist"innen die alltagspraktische „Definitionsmacht“ und Deutungshoheit über etliche dieser Fragen (Blankenburg/Feest 1972). Nicht selten gerät die nach wie vor überwiegend männlich geprägte (gendered) und immer noch recht homogene bzw. wenig diverse (Gewalt-)Organisation (vgl. Acker 1990; Wilz 2012, S. 123) in den Verdacht, bestimmten Differenzkategorien - wie z. B. Ethnizität - bei der Verrichtung ihrer Aufgabe übermäßige Bedeutung zuzumessen. Als prekäres polizeiliches „Erfahrungswissen“ halten (hegemoniale) und häufig auch medial verstärkte Vorstellungen davon, wer als Sicherheitsrisiko gelten kann, Einzug in die polizeiliche Praxis. Aus Sicht der von solchen Differenzierungsprozessen Betroffenen wird diese Versicherheitlichung häufig umgekehrt: die Polizei wird von ihnen explizit als (existentielle) Bedrohungsinstanz perzipiert. Es drängt sich die Frage auf, wessen Sicherheit die Polizei eigentlich gewährleistet (vgl. Loick 2018, S. 23-26).

Eine kritische sicherheitshistorische Analyse der Polizei sollte, so möchte ich im Folgenden argumentieren, diesem widersprüchlichen Doppelcharakter der Organisation und der in ihrem Auftrag handelnden Akteure stärker als bisher Rechnung tragen. Polizeiliche (Un-)Sicherheitsproduktion ist immer auch doing difference (West/Fenstermaker 1995): Als Normierungsinstanz und Differenzmaschine entwickelte die moderne Polizei bereits seit ihrer Entstehung in den (National-)Staaten des 19. Jahrhunderts innerhalb der Organisation wie auch nach außen spezifische, auf Differenzkonstruktionen basierende sicherheitspolitische Strategien, Sicherheitsentwürfe und Sicherheitspraktiken. Diese lassen sich wiederum in ihrer jeweiligen historischen Gewordenheit und Wandelbarkeit zum zeithistorischen Untersuchungsgegenstand machen. Dies gilt ebenso für Differenzkategorien, denen innerhalb der Organisation eine bedeutsame Rolle zukam, etwa die Frage der Integration von Frauen in den (Schutz-)Polizeidienst in der Bundesrepublik seit den ausgehenden 1970er Jahren, aber auch die kontroversen Diskussionen über ethnische Diversität in der Polizei seit den 1980er und 1990er Jahren. Diese Debatten drehten sich verstärkt um die Frage, wer das staatliche Gewaltmonopol repräsentieren solle und auf welche Weise dies zu geschehen habe.

Dieser Beitrag möchte den hier umrissenen Problemhorizont von Polizei, Sicherheit und Differenz ausleuchten und für eine erweiterte Perspektive auf die bundesdeutsche Polizeigeschichte werben. Untersuchungsgegenstand ist die West-Berliner Polizei während des „Kalten Krieges“ - bisweilen werden zeitlich wie räumlich diese Grenzmarkierungen überschrit- 
ten. Die wechselhafte und ereignisreiche Geschichte des Verhältnisses von Polizei und Gesellschaft soll an Fragen nach Differenzierungsprozessen und dem Wandel polizeilicher Sicherheitspraktiken rückgebunden werden. Ich beschränke mich in meinen Ausführungen auf die Schutzpolizei, also den in der Regel uniformierten, öffentlich unmittelbar wahrnehmbaren Teil der Polizeikräfte. Politische wie soziokulturelle Wandlungsprozesse zeitigten im Untersuchungszeitraum immer auch polizeiinterne Veränderungen, beide können folglich nicht getrennt voneinander betrachtet werden (vgl. Weinhauer 2003, S. 22). Dieser Text verknüpft daher analytisch beide Ebenen, die systematisch anhand der Differenzkategorien gender, racelethnicity und sexuality gegliedert werden. Im ersten Abschnitt werden Geschlechterbilder und -verhältnisse in und außerhalb der Polizei thematisiert. Ein zweites Kapitel widmet sich dem Verhältnis von Polizei und „Ausländern“ im sich rapide diversifizierenden West-Berlin seit den 1970er Jahren. Der dritte Teil wirft ein Schlaglicht auf die Beziehung von Polizei und Homosexuellen in der Metropole. So wird der Blick einerseits auf das Innenleben der Polizei gerichtet und der langsame Wandel von einer (fast ausschließlich) männlichen, militaristischen und homogenen (weißen) Truppe zu einer sich zaghaft diversifizierenden Organisation nachgezeichnet. Andererseits steht das Verhältnis von Polizei und Gesellschaft bzw. polizeilichem „Publikum“ im Mittelpunkt. Hierbei geht es insbesondere um den Differenz-erzeugenden Blick von Polizist*innen auf gesellschaftliche Devianz und die Verknüpfung eben jener Differenzkategorien mit alltäglichen Sicherheitsaspekten. Abschließend werden die Befunde zusammengefasst und unter der Fragestellung diskutiert, welchen heuristischen Mehrwert Differenz als Analysekategorie für die Polizeigeschichte bieten kann.

\section{Polizei und Geschlecht: Zwischen soldatischen Männlichkeitsidealen und "weiblichem Charme"}

Als im Februar 1952 die ersten Dienstanwärter für die neuaufgestellte Bereitschaftspolizei ihre Unterkünfte in Berlin-Heiligensee bezogen, hatten sich die jungen Männer im Alter von 19 bis 22 Jahren für eine insgesamt siebenjährige Dienstzeit verpflichtet, bevor sie in den alltäglichen polizeilichen Einzeldienst eintreten konnten - inklusive einer sogenannten „Zölibatsklausel“, die eine Heirat bis zum Ende der Dienstzeit verbot. Der Dienstalltag war militärisch geprägt, die Anwärter lebten kaserniert, und unter den Ausbildern fanden sich ehemalige Wehrmachtsoffiziere, die großen Wert auf Drill und Gehorsam legten, den sie mit häufig schikanierenden Praktiken durchzusetzen gedachten. $\mathrm{Zu}$ den Ausbildungsinhalten ge- 
hörten neben dem obligatorischen Formaldienst auch die Schulung an schweren Waffen wie Maschinengewehren und Granatwerfern (vgl. Steinborn/Krüger 1993, S. 93-97). Darin unterschied sich die Bereitschaftspolizei in West-Berlin kaum von den ebenfalls neu formierten Polizeikräften der westdeutschen Bundesländer und der kasernierten Volkspolizei in der unmittelbar benachbarten DDR (vgl. Fürmetz et al. 2001; Lindenberger 2003; Weinhauer 2003, S. 168-190; Dams 2008, S.11f.). Dennoch schlug sich die besondere Lage West-Berlins als „Vorposten der Freiheit“, wie es Ernst Reuter im September 1948 in seiner Rede vor dem Reichstag formuliert hatte, in einer höheren Intensität des kultivierten männlich-soldatischen Gemeinschaftsgefüges nieder, das über die Grenzen Berlins hinaus für Aufsehen sorgte. Zu den Kritikern der harschen Ausbildungspraxis gehörte beispielsweise der junge Gewerkschaftssekretär Klaus Hübner, der einige Jahre später Polizeipräsident in der Halbstadt werden sollte (vgl. Hübner 1952).

Der Historiker Klaus Weinhauer hat auf die zentrale Bedeutung der kasernierten Ausbildung für die Tradierung und (Re-)Aktualisierung von Erfahrungswissen, Mythen, Erzählungen und Ritualen in der Polizei hingewiesen. Aus geschlechtergeschichtlicher Perspektive hat er zudem die Spezifik der dort vermittelten „männerbündischen Gemeinschaftskultur“ (Weinhauer 2008, S.33) und damit einhergehender Ordnungsvorstellungen betont, die gleichermaßen Einfluss auf polizeiliche Einsatzpraktiken genommen hätten (vgl. ebd., S. 167-210). Mit dem Polizeiwissenschaftler Rafael Behr ließe sich die hegemoniale Männlichkeitsvorstellung der Cop Culture dieser Zeit idealtypisch als wehrhafte „Krieger-Männlichkeit“ fassen (Behr 2017, S. 543ff.; s.a. Behr 2008), die in West-Berlin auch im Rahmen eindrucksvoller Polizeifeste, der sogenannten "Großen Polizeischau“" im Berliner Olympiastadion, öffentlichkeitswirksam und gleichsam rituell inszeniert wurde (vgl. Schmeer 2020, S. 303-305). Während diese noch bis Mitte der 1960er Jahre martialisch anmutenden Aufführungen des staatlichen Schutzversprechens und polizeilicher Leistungsfähigkeit in einer von kommunistisch-sowjetischen „Feinden“ umzingelten Inselstadt einen symbolischen Schulterschluss mit der West-Berliner (Mehrheits-)Gesellschaft demonstrierten, bargen männliche Polizistenkultur, obrigkeitsstaatliche Vorstellungen von „Ruhe und Ordnung“ und militärische Einsatzkonzeptionen bei der Konfrontation mit jugendlichen und studentischen Protesten gewaltiges Konfliktpotential. Die zeithistorische Polizeiforschung hat dem Zusammenhang von Polizei, juveniler Devianz und (Jugend-)Protest im Vorfeld von „1968“, insbesondere in den 1950er und 1960er Jahren, bereits große Aufmerksamkeit gewidmet (vgl. exemplarisch Fürmetz 2006; Mecking 2020; Weinhauer 2001). Gleichwohl sei kurz darauf verwiesen, 
wie persistent die hier geschilderten Männlichkeitsvorstellungen waren und wie widerständig sich die Organisationskultur der Polizei gegen einschlägige Reformimpulse erwies. Dies zeigte sich in West-Berlin zum Beispiel in der zunächst scharfen Ablehnung der Erprobung neuer Einsatztaktiken durch eine Mehrheit der Schutzpolizeitruppe. Ein im Jahr 1969 aufgestelltes „Diskussionskommando“ zur kommunikativen Entschärfung des studentischen Protestgeschehens wurde von Kollegen nach ersten Einsatzerfahrungen intern abschätzig als „Kapitulationsgruppe“ bezeichnet. Deeskalation scheint, im Gegensatz zur notfalls gewaltsamen Lösung, ganz offenbar als Infragestellung zupackend-maskuliner Polizeiarbeit wahrgenommen worden zu sein (vgl. Kleinknecht/Sturm 2004, S. 211f.; Schmeer 2020, S. 311). Dass derartige Vorstellungen auch den (mehrheits-)gesellschaftlichen Blick auf die Polizei prägten, lässt die Debatte um sogenannte „HaarErlasse“ in den frühen 1970ern erahnen. Teile der Polizeiführung bundesweit befürchteten einen Imageschaden der Behörde durch „langgelockt[e] Schupos", die in West-Berlin von Bürger"innen auch mal ,herabwürdigend“ als „Fräulein“ oder „uniformiert[e] Hippie[s]“ bezeichnet wurden (Der Spiegel 1972). So ließ offenkundig auch die gesellschaftliche Vorstellung davon, wie ein ,ordentlicher' Schutzmann auszusehen habe, keinen großen Spielraum für Abweichungen oder weibliche Konnotationen.

Die hier skizzierte, von einer ausgeprägten „Krieger-Männlichkeit“ geprägte Cop Culture verleitet indes allzu leicht dazu, die Geschichte von Frauen in der Polizei zu übersehen. Für die „Anfänge weiblicher Polizei in Deutschland“" (Nienhaus 1999; Götting 2010) hat die Historikerin Bettina Blum die von Polizistinnen selbst(-bewusst) behauptete Geschlechterdifferenz herausgearbeitet, die unter dem Stichwort einer „weiblichen Eigenart" als Eintrittskarte in den Dienst des staatlichen Gewaltmonopols seit der Weimarer Republik fungiert hatte. Frauen hatten in der „Weiblichen Kriminalpolizei“ (WKP) vor allem sozial-fürsorgerische Aufgaben übernommen und damit ein mütterliches Bild weiblicher Polizeiarbeit geprägt. Nach 1933 sei dieser soziale Charakter durch eine vorauseilende Anpassung an die repressiven (Gewalt-)Strukturen des Nationalsozialismus weitgehend verloren gegangen (vgl. Blum 2012, S.381-391). Die Beamtinnen der WKP wurden auch aus Gründen der professionellen Selbsterhaltung zu willigen Mittäterinnen der Verfolgung von durch die Nationalsozialisten als „gemeinschaftsfremd“ oder „asozial“ bezeichneten (weiblichen) Jugendlichen, etwa in den sogenannten "Jugendschutzlagern“ (vgl. Götting 2009, S. 505-509). In der Nachkriegszeit konnten weibliche Polizistinnen an Traditionsbestände und Dienstauffassungen sowohl aus Weimar als auch des Dritten Reiches anknüpfen (vgl. Blum 2015). 
Auch in Berlin bestand die WKP nach der Kapitulation fort. Vor allem auf Betreiben der Sowjets und Briten diskutierten die alliierten Besatzungsbehörden bereits ab Mitte 1945 den zusätzlichen Einsatz von Frauen im Schutzpolizeidienst. Gegen massive „Abwehrbemühungen der Männer gegen die drohende weibliche Konkurrenz" setzten die Alliierten im Verlauf des Jahres 1946 die Einstellung der ersten Schutzpolizistinnen durch (Nienhaus 2001, S. 144). Diese wurden im Volksmund aufgrund der amtlichen Abkürzung für die „Weibliche Schutzpolizei“ (WSP) bald als „Wespen" bezeichnet (Der Abend 1968). In einem Schreiben des Polizeipräsidiums an das zuständige Subkomitee des Public Safety Committee der damals noch ungeteilten Alliierten Stadtkommandatur wurde eine grundsätzliche Gleichstellung männlicher und weiblicher Polizeikräfte festgeschrieben, wenngleich es in einigen Punkten sogleich Einschränkungen gab: So sollten die Polizistinnen nicht für Aufgaben eingesetzt werden, die „das Ansehen der Frau in der Oeffentlichkeit“ herabsetzen würden. Weitere „besondere Aufgabengebiete" umfassten als weiblich imaginierte soziale Tätigkeiten wie die „Überwachung der Kinder und Jugendlichen, insbesondere der weiblichen“. Die Uniform war derjenigen der männlichen Kollegen zwar angepasst, allerdings war ein halblanger Rock statt einer ,unweiblichen Hose vorgeschrieben. Die neuen Ordnungshüterinnen sollten auch bewaffnet werden. Als Dienstpistole kam indes nur eine kleinere Variante infrage, da "die weibliche Hand sich nicht für die Handhabung von Schußwaffen größeren Kalibers“ eigne. Auch die Ausrüstung mit einem Polizeiknüppel war nicht vorgesehen. Die Schutzpolizistinnen seien, so urteilten die Regulatoren im Polizeipräsidium, für die „Brechung von Widerstand durch Anwendung von Gewaltmitteln im allgemeinen " nicht geeignet. ${ }^{2}$

Nach der Spaltung der Berliner Polizei im Zuge der wachsenden Spannungen zwischen den Westmächten und der Sowjetunion im Juli 1948 wurden nur noch einige wenige Polizistinnen im Schutzpolizeidienst der Westbezirke eingestellt. Dies hing auch mit einer sich entspannenden (männlichen) Personalsituation durch zurückkehrende Kriegsgefangene zusammen. Auf der Führungsebene wurden bei den Frauen vergleichsweise höhere Ausfallzeiten moniert und allgemein die Eignung zum Schutzpolizeidienst zunehmend in Frage gestellt (vgl. Nienhaus 2001, S. 147f.). Die Schutzpolizistinnen fanden sich in der Folge zunehmend in den In-

2 Vgl. „Schreiben Markgraf an Sub-Committee des Public Safety Committees der Alliierten Stadtkommandatur (Bestimmungen über die weibliche Polizei)“, 23. April 1946. In: Landesarchiv Berlin (LAB), C Rep. 303-09, Nr. 073, Bl. 8-15. Vgl. dazu auch Nienhaus 2001, S. 140-152. 
nendienst versetzt. 1951 waren noch 167 „Wespen“ bei der West-Berliner Polizei beschäftigt, von denen einige in die WKP wechselten, während andere ihren Posten für eine traditionell weiblich konnotierte Tätigkeit als Schreibkraft aufgaben (ebd., S. 150). Knapp zwei Jahrzehnte später, im Oktober 1968, galten die Schutzpolizistinnen im Westteil der Stadt als aussterbende Art. Die zu diesem Zeitpunkt verbliebenen 53 Ordnungshüterinnen sollten nun vollends in die weibliche Kriminalpolizei eingegliedert werden (vgl. Der Abend 1968). Die weibliche Schutzpolizei in West-Berlin konnte sich also nicht langfristig in der "gendered organization“ (Acker 1990; Weinhauer 2003, S. 14), der hegemonial-männlichen Organisationskultur etablieren; männlich-autoritäre Strukturen blieben dominant. Auch in der DDR geriet die Vorstellung einer „weiblichen Eigenart“ aufgrund der Fokussierung auf die Kategorie „Klasse“ und vor dem Hintergrund des sich intensivierenden Ost-West-Konflikts und einer umfassenden Militarisierung der DDR-Polizei zunehmend in den Hintergrund (vgl. Blum 2012, S. 383f.).

Seit Mai 1971 wurden im Westen zur Überwachung des ruhenden Verkehrs und als Auskunftspersonen erneut Frauen in der Polizei eingesetzt. Die als „Politessen“ oder „PAngSODs“ (Polizei-Angestellte im Sicherheitsund Ordnungsdienst) bekannt gewordenen Ordnungshüterinnen sollten als „Brigade des Charmes“ (Beil 1968) die männlichen Kollegen entlasten und die unbeliebte Aufgabe übernehmen, gegen renitente Parksünder vorzugehen. Die Polizeiführung versprach sich vom Einsatz der jungen Frauen trotz der potentiell undankbaren Aufgabenbereiche auch einen Imagegewinn, den die umstrittene Organisation nach den Turbulenzen und Gewalteskalationen im Zuge der Studierendenproteste dringend brauchte (vgl. Schmeer 2020, S. 305-308). Es dauerte allerdings noch bis 1978, bis Frauen erneut der Weg in den regulären Schutzpolizeidienst geebnet wurde. West-Berlin nahm hier in der Bundesrepublik eine Vorreiterrolle ein, wenngleich die Debatte um Frauen in der Polizei länderübergreifend geführt wurde und im Laufe der 1980er Jahre die meisten Bundesländer nachzogen. Einzig in Bayern verzögerte sich die Aufnahme von Frauen in die Schutzpolizei bis Anfang 1990 (vgl. Tielemann 1993).

West-Berlins Polizeivizepräsident Gerhard Pfennig legte 1978 seine Auffassung über die Stellung der Frau in der Polizei ausführlich dar und markierte das Vorhaben als ein (geschlechter-)politisches Projekt: Er betonte, dass es ihm vor allem um die Stärkung der „Emanzipation der Frau“ ginge. Gleichzeitig verstand er seinen Ansatz als direkte Reaktion auf „das Phänomen des überaus starken weiblichen Anteils an der Terroristenszene“, dem er allerdings keine geschlechtsspezifische Reaktion der Polizei entgegenhalten wollte. Neben den von der neuen, im Gefolge von „1968“ entste- 
henden Frauenbewegung angestoßenen Debatten scheint auch der radikale Bruch der RAF-Terroristinnen mit traditionellen Geschlechterrollen die Vorstellung von (bewaffneten) Frauen im Schutzpolizeidienst maßgeblich befördert zu haben (vgl. Pfennig 1978, S. 40; zu Frauen in der RAF vgl. Diewald-Kerkmann 2009). Pfennig gelangte zu der Einschätzung, dass „Probleme des Geschlechts“ einer Verwendung von Frauen im Schutzpolizeidienst jedenfalls nicht (mehr) entgegenstünden. Neue, auf Frauen zugeschnittene Einsatzformen könnten darüber hinaus zu einer Verbesserung des polizeilichen Images beitragen. Gleichzeitig müssten aber auch „Vorurteile gegen den Einsatz von Frauen" im Polizeidienst intern abgebaut werden, was sich ganz explizit sowohl auf das Innenleben der Organisation als auch auf deren Verhältnis zum polizeilichen Publikum bezog (Pfennig 1978, S. 42f.).

Insbesondere in der Außendarstellung der Polizei stand zunächst nach wie vor die Betonung einer spezifisch weiblichen Dienstauffassung im Vordergrund. West-Berlins Innensenator Peter Ulrich (SPD) wurde bereits vor Dienstantritt der ersten fertig ausgebildeten 24 Schutzpolizistinnen (allesamt umgeschulte „Politessen“ im Alter von 27 bis 39 Jahren) zum 1. September 1980 in den Medien mit den Worten zitiert, dass die neuen Kolleginnen keineswegs als herumabenteuernde „Karate-Emmas“ zu betrachten seien. Stattdessen, versicherte er, würden die „engagierte[n] Frauen“ ihren Dienst „mit Charme“ ausüben (Neurath 1980). Ulrich nahm damit Bezug auf eine populäre Fernsehserie - das Bild der modernen und ebenso schlagfertigen wie attraktiv-charmanten Frau in der Person der weiblichen Hauptfigur „Emma“ avancierte zu dieser Zeit zu einer beliebten Umschreibung für Polizeibeamtinnen in den Medien (Blum 2012, S. 335-339; Blum 2015, S. 124f.). Obwohl etwa die Süddeutsche Zeitung darauf verwies, dass die Polizistinnen grundsätzlich das gleiche Aufgabenspektrum abdeckten wie ihre männlichen Kollegen, waren schwere Waffen wie Maschinengewehre oder gar Handgranaten, die damals noch zum selbstverständlichen Inventar der Behörde gehörten, nicht Teil ihrer Ausbildung. Auch bei Demonstrationen sollten die jungen Polizistinnen „allenfalls im zweiten Glied" stehen. Der Bericht schloss mit der Feststellung, die Polizei hoffe, dass "die Herren Autofahrer sich nicht allzusehr beim Blick nach den weiblichen Verkehrspolizisten den Hals verdrehen und dem Vordermann drauffahren“"würden (Süddeutsche Zeitung 1980).

Die neuerliche Einführung einer weiblichen Schutzpolizei in West-Berlin Ende der 1970er-Jahre war von launig-sexistischen Tönen begleitet, die vielfach auf das Äußere der Beamtinnen zielten. In einer Abfolge von Diminutiven befleißigte sich beispielsweise die Berliner Morgenpost im Sommer 1980, das neue Aufgabenfeld der wegen der Uniformen sogenannten 
„weißen Mäuschen“ zu beschreiben: Diese würden künftig den Verkehr „mit charmantem Wink“ regulieren und dabei „ein kesses grünes Polizeimützchen auf dem Blondschopf" tragen. Immerhin wurde anerkennend konstatiert, dass elf der Frauen in der Abschlussprüfung besser abgeschnitten hätten als ihre 138 männlichen Kollegen (vgl. Bentheim 1980). Trotz aller Gleichstellungs- und Fortschrittssemantiken, die in den Quellen dieser Zeit auftauchen: Der Topos der „,weiblichen Eigenart“ scheint auch bei dieser zweiten Öffnung der Schutzpolizei für Frauen diskursiv wirkmächtiger gewesen zu sein.

Die Öffnung des Schutzpolizeidienstes für Frauen war wiederum eine Reaktion auf ein drängendes Nachwuchsproblem der West-Berliner Ordnungshüter (vgl. Weis 1980). Allein für das Jahr 1980 wurde laut Medienberichten mit 200 bis 250 unbesetzten Stellen gerechnet. So erklärt sich, dass ab Herbst 1980 nach einer intensiven medialen Anwerbekampagne der Zugang zum mittleren Schutzpolizeidienst auch für Frauen möglich wurde, die zuvor nicht als „Politessen“ tätig gewesen waren. Insgesamt bewarben sich bis November 1980 rund 500 junge Frauen, eingestellt wurden 88 Dienstanfängerinnen. Für das Jahr 1981 wurde mit weiteren 64 Einstellungen gerechnet (vgl. Polizeischau 1980, S.4). Die Anzahl eingestellter Polizistinnen nahm im Verhältnis zu ihren männlichen Kollegen in den Folgejahren immer stärker zu, wenngleich das Einstellungsverhältnis bis Ende der 1980er Jahre vielfach negativ quotiert war (vgl. Tielemann 1993, S. 19-21). Auch in der Praxis und organisationsintern scheint die Integration von Frauen in den Schutzpolizeidienst keineswegs reibungslos verlaufen zu sein. So mussten sich die Ordnungshüterinnen gegen die offenkundigen Vorurteile ihrer Kollegen durchsetzen und forderten Gleichberechtigung beispielsweise in den Polizeigewerkschaften ein (vgl. Der Tagesspiegel 1982b; Grun 1982, S.71). Ob die Teilhabe von Frauen das (männliche) Antlitz und Sicherheitshandeln der Polizei verändert und damit einen (langen) „Abschied von der Männlichkeit" (Dams 2008, S. 14) befördert hat, steht indes zu bezweifeln. Gegenwärtig ist eher von einer „Renaissance aggressiver Maskulinität“ (Behr 2018) in der Polizei die Rede.

\section{Polizei und Ethnizität: Umgang mit „Ausländern" und die Anfänge von "Diversität in Uniform"}

Das Verhältnis von Polizei und „Ausländern“ und die Bedeutung der Differenzkategorie race bzw. ethnicity wurden in der deutschen Polizeigeschichtsschreibung bislang noch nicht systematisch erforscht, obgleich die sozialwissenschaftliche Polizeiforschung das Thema durchaus aufgegriffen 
hat (vgl. Hunold et al. 2010). Dieser Befund verwundert insbesondere mit Blick auf West-Berlin: Die Demographie der Inselstadt hatte sich seit den späten 1960er-Jahren auch aufgrund des Zuzugs von "Gastarbeiter"innen“ rasant verändert. Vor allem durch den Familiennachzug, der nach dem Anwerbestopp im November 1973 stark an Bedeutung gewann, nahm die Zahl insbesondere von türkeistämmigen Menschen stetig zu. ${ }^{3}$ Für die politischen Entscheidungsträger und die Polizeiführung West-Berlins stellte das sowohl in sozialer und fürsorglicher als auch in sicherheitlicher Hinsicht eine große Herausforderung dar. Beide Instanzen reagierten in unterschiedlicher Weise auf die gesellschaftlichen Veränderungsdynamiken, und sie bedienten sich dabei vielgestaltiger Differenzzuschreibungen. Prägend war eine „Wahrnehmung der Migrant"innen als kulturell ,andere‘, deren Anwesenheit die Stabilität der Stadtgesellschaft gefährdete" (Borgmann 2019, S. 294). Um der Bildung von sogenannten „Ausländer-Gettos“ (so z.B. Der Spiegel 1974) zuvorzukommen, beschloss der SPD-geführte West-Berliner Senat ab 1975 eine hochumstrittene „Zuzugssperre“ für Bezirke mit einem hohen Anteil an Migrant*innen, namentlich für Kreuzberg, Tiergarten und Wedding. Die Regulierung und Reduzierung des „legalen Gastarbeiter-Zustroms“ sowie die entschiedene Bekämpfung des Zuzugs von „Illegalen“ waren die handlungsleitenden Motive der Polizei im Umgang mit „Ausländern“ (Polizeischau 1975, S. 4; vgl. auch Haibach 1979). Vor allem für den letztgenannten Zweck hatte die Polizei in der geteilten Stadt bereits 1971 die unter dem Akronym „AGA“ firmierende Sondereinheit "Arbeitsgebiet gezielte Ausländerüberwachung" eingerichtet, die später in „Arbeitsgruppe Ausländer“ umbenannt wurde (Diederichs 1993, S. 46). Ausgangspunkt für diesen neuen Tätigkeitsbereich war die Polizeiinspektion im Wedding mit insgesamt drei Beamten der Schutzpolizei. Als „erster Berliner Sachverständiger der Polizei für Türkenfragen“ galt der dort seinen Dienst verrichtende Polizeihauptmeister Hans-Joachim Jankus. In einem Artikel für die Polizeischau, der Zeitschrift der Berliner Polizei, wurde er als ausgewiesener Experte für den polizeilichen Blick auf die gleichsam exotisierten ,Anderen' charakterisiert, dessen „fehlerfreies Türkisch und das Auskennen in den Sitten und Gebräuchen der Muselmanen" ihm den Spitznamen "Groß-Mufti vom Wedding" eingebracht

3 Im Jahr 1975 waren rund 85.000 Türkeistämmige und etwa 30.000 Jugoslawen in West-Berlin gemeldet, die damit die Mehrheit der insgesamt gut 185.000 „Ausländer" bei einer Gesamtbevölkerung von rund zwei Millionen stellten (Statistisches Landesamt Berlin 1976, S. 33-35). 
hätten. Der Autor ließ offen, von wem Jankus so genannt wurde (Polizeischau 1975 , S. 4).

1975, so schildert der Artikel weiter, waren bereits 32 erfahrene Beamte in der AGA tätig, die regelmäßig türkische und/oder serbokroatische Sprachkurse besuchten, um sich mit ihrem polizeilichen Gegenüber besser verständigen zu können. Das Hauptziel der AGA bestand darin, die kommunikative wie kulturelle „Diskrepanz zwischen Ausländern und Polizei“ abzubauen und dabei nicht ausschließlich kontrollierend-repressiv, sondern gleichzeitig erzieherisch-sozialfürsorgerisch aufzutreten (Polizeischau 1975, S. 4). Diese neuen kommunikationsorientierten Einsatzkonzeptionen sind als Folge der nach 1967/68 einsetzenden organisatorischen wie kulturellen Reformprozesse (nicht nur) in der West-Berliner Polizei zu verstehen, die in den 1970er Jahren unter anderem eine stärkere rechtsstaatliche Orientierung und ein Streben nach mehr „Bürgernähe“ zeitigten (vgl. Mecking 2020; Schmeer 2020; Weinhauer 2020, S. 180-182). Dem Auftrag der AGA lag eine weitere Differenzkonstruktion zugrunde, die wiederum die Gruppe der „Ausländer“ in sich mit legalem Aufenthaltsstatus in WestBerlin und der Bundesrepublik aufhaltende Menschen und sogenannte „Illegale" unterteilte. Letztere wurden mit der affektgeladenen Bedrohungsmetapher eines „Strom[s]“ bedacht, der sich „nicht drosseln“ lasse, weil viele der illegal Einreisenden in der Bundesrepublik ,immer noch das ,gelobte Land"“ sähen (Polizeischau 1975, S. 5). In der exotisierenden Beschreibung der polizeilichen „Ausländerüberwachung" scheint bisweilen auch Mitgefühl oder Verständnis für die oftmals prekären Lebensbedingungen der Zugezogenen durch (ebd.). In der „Doppelfunktion“ (Diederichs 1993) von Vertrauenswerbung und Repression offenbart sich gleichwohl die generelle Aporie polizeilicher Sicherheits- und Bedrohungsproduktionen.

Besonders virulent wurde die Frage, wen die Polizei eigentlich vor wem schütze - und welche Gruppen gerade nicht - im polizeilichen Umgang mit Asylsuchenden ab den späten 1970er Jahren. Hier zeigen sich explizit rassistische ${ }^{4}$ Differenzierungen, nicht nur im Blick auf das polizeiliche Gegenüber, sondern auch in der konkreten polizeilichen Praxis. Der Anstieg

4 Rassismus wird hier nach dem Definitionsversuch von Birgit Rommelspacher als machtlegitimierendes und -reproduzierendes "System von Diskursen und Praxen“ verstanden, in welchem „soziale und kulturelle Differenzen naturalisiert“, Gruppen von Menschen homogenisiert, als „grundsätzlich verschieden und unvereinbar gegenübergestellt" und schlussendlich auch hierarchisiert werden (Rommelspacher 2009, S. 29). 
der Zahl asylsuchender Personen zwischen 1975 und $1980^{5}$ hatte nicht nur eine kontroverse, fast schon hysterisch geführte Debatte über eine Reform des bundesdeutschen Asylrechts zur Folge (vgl. Herbert 2001, S. 263-285; Münch 1993, S. 38-110), sondern rückte auch West-Berlin als vermeintlich unreguliertes „Schlupfloch“ für ,illegale“ Einwanderung in den Fokus des politischen Interesses. Gerade die Nichtanerkennung der Berliner Mauer als reguläre völkerrechtliche Staatsgrenze und die damit begründeten ausbleibenden Kontrollen im Personenverkehr auf West-Berliner Seite führten dazu, dass über Ost-Berlin viele Asylsuchende einreisten. Um der Doktrin treu zu bleiben und dennoch das sogenannte „Berliner Loch“ polizeilich in den Griff zu bekommen, bediente sich die West-Berliner Polizei und vor allem die AGA der damals noch nicht so bezeichneten Praxis des racial profiling (vgl. Stokes 2020). Die Asylsuchenden (vor allem aus sogenannten „Entwicklungsländern“) reisten nach der Ankunft am Ost-Berliner Flughafen Schönefeld mit einem Transitvisum weiter nach West-Berlin. Entsprechend kontrollierten Beamte der AGA die einschlägigen S- und U-Bahn-Verbindungen regelmäßig nach sogenannten „Illegalen“. Die Auswahlkriterien für eine Personenkontrolle beschränkten sich dabei häufig auf das äußere Erscheinungsbild, sodass auch Menschen mit „legalem“ Aufenthaltsstatus von der Polizei herausgegriffen wurden. 1978 fahndete die Schutzpolizei erstmals auf den in West-Berlin liegenden S-Bahnhöfen Gesundbrunnen und Lehrter Bahnhof nach aus Ost-Berlin einreisenden Pakistaner*innen (vgl. Süddeutsche Zeitung 1978). Auch im Fall der Uund S-Bahn-Kontrollen nahm die Polizei offenkundig weitere Binnendifferenzierungen zwischen den als fremd oder „illegal“ gelesenen Menschen vor. Im West-Berliner Tagesspiegel berichtete der oben bereits erwähnte AGA-Beamte Jankus 1982 vom Arbeitsalltag und den Erfahrungen seiner Kollegen. Insbesondere ging er auf die Kontrolle von „Schwarzafrikaner[n]“ ein und bekräftigte Ressentiments gegenüber Schwarzen Menschen. Diese würden sich ihr „ähnliches Aussehen zunutze“ machen und untereinander Pässe tauschen, um einen legalen Aufenthaltsstatus vorzutäuschen. Insbesondere seien sie bei Kontrollen im Dunkeln „nur schwer auseinanderzuhalten“ (Der Tagesspiegel 1982a). Er gab damit zu verstehen,

5 Seit 1975 war die Zahl asylsuchender Personen in der BRD stark angestiegen. Während in diesem Jahr noch 9.627 Menschen einen Asylantrag stellten, stieg diese Zahl beschleunigt ab 1977 und bis 1980 auf über 100.000 (Münch 1993, S. 253). 
dass in der Logik der Polizei Hautfarbe und damit verknüpfte Stereotype als spezifisches Sicherheitsproblem wahrgenommen wurden. ${ }^{6}$

Gleichwohl regte sich auch verhaltener gesellschaftlicher Protest gegen diese Praxis, wie aus kritischen Zuschriften an das Polizeipräsidium hervorgeht. Als in der Folge des Sprengstoffanschlags auf die Diskothek La Belle in Friedenau 1986 verstärkt nach Terroristen gefahndet und dabei etliche Menschen aufgrund ihrer Hautfarbe oder anderer ethnischer $\mathrm{Zu}$ schreibungen in Polizeikontrollen gerieten, kritisierten einige Eingaben das Vorgehen der Polizei. Die „Interessengemeinschaft der mit Ausländern verheirateten Frauen" forderte den sofortigen Stopp der Praxis eines speziellen „Einsatzkommandos zur Kontrolle der Schwarzköpfe“. Eine Eingabe der prominenten Filmregisseurin Helma Sanders-Brahms schildert die harsche polizeiliche Kontrolle einer türkischen Familie, wogegen die Verfasserin „mit eher unfreundlichen Grüßen“ empört protestierte. ${ }^{7}$ Auch andere Tätigkeiten der AGA wie die intime Kontrolle von unter dem Verdacht der "Scheinehe“ stehenden Lebensgemeinschaften von binationalen Paaren gerieten in den Folgejahren zunehmend in die Kritik (vgl. Blumenthal/Fritz 1984; Diederichs 1986).

Auch innerhalb der politischen Führung West-Berlins und in internen Debatten der Polizei wurde intensiv über eine personelle Diversifizierung der Polizei nachgedacht, um der als dringlich empfundenen Herausforderung durch „Ausländer“ entgegenzuwirken. Mitten in die bereits skizzierte Debatte um das Asylrecht platzte der Vorschlag des Berliner SPD-Innensenators Peter Ulrich, künftig auch türkeistämmige Bürger West-Berlins in den Polizeidienst aufzunehmen. Er begründete dies mit der wichtigen Signalwirkung insbesondere für die jüngeren der rund 100.000 Türken in der Halbstadt (Der Spiegel 1979a). Diese symbolträchtige Zahl stand stellvertretend für den Höhepunkt der rasanten gesellschaftlichen Veränderungsdynamik in West-Berlin, die sich seit Ende der 1960er Jahre vollzogen hatte und von der Presse mit Faszination und Sorge begleitet worden war.

Ulrich schlug im Spiegel-Interview in dieselbe Kerbe wie der bereits zitierte Artikel aus der Polizeischau: Mit der merklichen Differenzierung der

6 Insgesamt hätten die Beamten der AGA 1984 ca. 70.000 „Ausländer“ kontrolliert und dabei rund 9.000 Personen wegen eines festgestellten Verstoßes gegen das Ausländergesetz festgenommen (Gaserow 1985).

7 Siehe dazu die entsprechenden Schreiben in LAB Berlin B Rep. 004, Nr. 3655 : „Helma Sanders-Brahms an den Herrn Innensenator der Stadt Berlin“, Schreiben vom 13.04.1986; „Interessengemeinschaft der mit Ausländern verheirateten Frauen e.V. an den Senator für Inneres“, Schreiben vom 26.05.1986. 
West-Berliner Gesellschaft, die sich freilich nicht nur auf Menschen türkischer Herkunft beschränkte, gingen "spezifische Sicherheitsprobleme“ einher, welche die Polizei vor neue Probleme stelle. Dabei sei es essenziell, die „kulturellen Hintergründe, die Sprache [zu] kennen und alles, was damit zusammenhängt“. Der Innensenator verwies damit ganz explizit auf eine hegemonial-differenzielle Sicherheitslogik von Ordnungshütern und Sicherheitspolitikern. Die „Ausländer-Kriminalität“ sei zwar nicht besonders hoch, aber dennoch ,andersartig“: Ulrich zählte dazu spezifische Deliktformen wie Messerstechereien oder „Auseinandersetzungen in Familienbereichen, die für uns nicht so ganz nachvollziehbar" seien (Der Spiegel 1979a).

Ganz ähnlich argumentierte 1981 in einem Vortrag der hochrangige Ordnungshüter und spätere Polizei-Vizepräsident Dieter Schenk, der einem englischsprachigen Publikum das West-Berliner Modell des sogenannten „Kontaktbereichsbeamten“ erläutern sollte. Diese im Zuge der großen Polizeireform 1973 eingeführte bürgernahe Praxis des community policing habe zwar eine Verbesserung des Verhältnisses von Polizei und Mehrheitsgesellschaft herbeigeführt; in Stadtvierteln mit überwiegend migrantischer Bevölkerung jedoch hätten die (weißen, männlichen und älteren) Beamten, die als freundlicher Schutzmann an der Ecke und Vertrauens- wie Ansprechperson der Bevölkerung dienen sollten, im Alltag empfindliche Kommunikations- und Akzeptanzprobleme. ${ }^{8}$ Aus Perspektive der Polizeiführung ergab es also durchaus Sinn, künftig auch türkeistämmige Polizist"innen zur Legitimitäts- und Akzeptanzsteigerung der Behörde einzusetzen. Gleichwohl ergab sich hier das beamtenrechtliche Problem, dass entsprechende Kandiat*innen vor einer Aufnahme in den Öffentlichen Dienst die deutsche Staatsangehörigkeit annehmen mussten. Allerdings sah diese Regelung durchaus Ausnahmen im Falle eines „dringende[n] dienstliche[n] Bedürfnis[ses]" vor. Bei „über 90000 Türken in dieser Stadt", so Innensenator Ulrich, liege dieser „Ausnahmetatbestand zweifellos vor“, wenngleich er direkt relativierte, dass „Türken, Jugoslawen oder Griechen“ in der Polizei auch zukünftig allenfalls Ausnahmen bleiben sollten (Ulrich 1979).

Die Interviewfragen der Spiegel-Redaktion zielten ausdrücklich auf das Spannungsverhältnis von Mehrheitsgesellschaft und Minderheit. So wurde etwa die Befürchtung geäußert, eine „fremde Mentalität" könnte mit den „ausländischen Ordnungshütern“ Einzug in Polizeikultur wie polizeiliche

8 Vortrag Schenk, Neighbourhood Policing in Berlin, 3.2.1981, in: Polizeihistorische Sammlung Berlin (PHS Berlin) 3.54.4 (Kontaktbereichsdienst). 
Praxis halten und künftig etwa „Messerstechen als Kavaliersdelikt" klassifiziert werden. Weiteres Konfliktpotential sah der Spiegel in der Frage der Akzeptanz der türkeistämmigen Polizisten durch alteingesessene Berliner*innen und einem möglichen Loyalitätszwiespalt zwischen dem Herkunftsland der Beamt*innen und dem deutschen Staat (vgl. Der Spiegel 1979a). An dieser Stelle scheinen die Debatte über die Integration der vormaligen "Gastarbeiter", die Frage nach ausländischen Beamten und die heftigen Kontroversen um das (west-)deutsche Einbürgerungsrecht durch (vgl. Herbert 2001, S. 231-285). Gleichermaßen wurde indes immer auch die Frage verhandelt, wer denn eigentlich Sicherheit für wen produzieren dürfe - und ob nicht bestimmte Gruppen, selbst im Polizeiberuf, eher ein Sicherheitsrisiko darstellten. Dass diese Diskussion tiefergehend auch die Frage nach der (Nicht-)Toleranz oder gar einem strukturellen Rassismus der Mehrheitsgesellschaft aufwarf, war im Problemhorizont einiger Zeitgenossen durchaus präsent. ${ }^{9}$ Ulrich schloss sein Spiegel-Interview mit den Worten: „Es geht - das muß man auch mal ganz öffentlich benennen - um das Problem: Dürfen Schwarze Weiße verhaften“ (Der Spiegel 1979a).

Während diese Debatte in vollem Gange war und sich Peter Ulrich der erbitterten Kritik der christdemokratischen Opposition sowie der Polizeigewerkschaften an einer „Türkenpolizei“ erwehren musste (vgl. Wüller 2010, S. 165-167), machte gegen Ende des Jahres 1979 die Nachricht die Runde, dass mit dem 20-jährigen Nihat Dogru bereits der erste türkeistämmige Mann in den Polizeidienst der Halbstadt eingetreten war. Dieser lebte seit seinem sechsten Lebensjahr in der Spree-Metropole und hatte zuvor zusätzlich zur türkischen die deutsche Staatsangehörigkeit angenommen (vgl. Weis 1979). Einige Leserbriefe an den Spiegel machen deutlich, wie sehr diese Tatsache die Leserschaft polarisierte. Eine offen rassistisch formulierte Zuschrift lautete etwa: „Ich würde niemals einen Kanaken in deutscher Uniform respektieren“ (Der Spiegel 1979b). Andere Leser*innen begrüßten das Novum in der Berliner Polizeigeschichte und sprachen dem jungen Polizeibeamten Mut für die kommenden Aufgaben zu (vgl. Der Spiegel 1979c). Die Intensität der Debatte stand indes in keinem Verhältnis zur äußerst geringen Anzahl der Polizeibeamten mit Migrationsgeschichte in der West-Berliner Polizei bis weit in die 1990er Jahre. Genaue Statistiken zu dieser Frage werden aufgrund des Gleichberechtigungs-

9 Die Begriffe „Rasse“ und „Rassismus“ tauchen in den Quellen nicht auf. Gleichwohl sei hier auf neuere Trends vor allem in der zeithistorischen deutschen Immigrationsforschung verwiesen, die auf die Kontinuität rassistischer Denkkonfigurationen in Deutschland, insbesondere in der Diskursfigur der „Ausländer“, hingewiesen hat (vgl. Alexopoulou 2019). 
grundsatzes nicht erfasst. Die Polizeiwissenschaftlerin Daniela Hunold stellt für 2009 beispielsweise einen Anteil von Polizist“innen mit „Migrationshintergrund" von 1,86 Prozent bei der Berliner Polizei fest. Beinahe dreißig Jahre zuvor dürfte dieser Anteil folglich verschwindend gering gewesen sein (vgl. Hunold et al. 2010, S. 138). Dies wird umso deutlicher, wenn man sich vergegenwärtigt, dass im Erfassungszeitraum 1945 bis 1980 nur insgesamt 350 Türkeistämmige eingebürgert wurden, 55 davon im Berichtsjahr (vgl. Statistisches Landesamt Berlin 1981, S. 43).

Am Streit um türkeistämmige Polizisten in West-Berlin lassen sich wie in einem Brennglas zentrale sicherheitskulturelle Topoi in einer sich diversifizierenden (Stadt-)Gesellschaft identifizieren, die zum Teil bis heute nachwirken (siehe dazu etwa Dudek 2009). Bei all diesen Topoi spielen race bzw. ethnicity als Differenzkategorien eine zentrale Rolle: Dies betrifft erstens die Frage, von welchen Akteuren das staatliche Gewaltmonopol in der Bundesrepublik überhaupt vertreten werden sollte. Ob „Ausländer“ oder BPoC die Ausübung von (polizeilichen) Hoheitsrechten wahrnehmen durften, war Gegenstand heftiger juristischer wie gesellschaftlicher Kontroversen. Und selbst in beamtenrechtlich „unproblematischen“ Fällen wurden von Kritiker*innen Loyalitätskonflikte, Parteinahme und Sicherheitsbedenken gegen migrantische Kolleg*innen ins Feld geführt und dies noch bis weit in die 2000er Jahre (vgl. Wüller 2010, S. 167f.). Zweitens wurde verhandelt, wen die Polizei eigentlich vor wem und mit welchen Mitteln zu beschützen habe. Wie das Beispiel der Fahndungspraxis der AGA zeigt, vollzog sich die Sicherheitsproduktion für die Mehrheitsgesellschaft vielfach zum Nachteil von Menschen, die aufgrund äußerer Merkmale von der Polizei als „nicht deutsch“ gelesen und mithin als Risiko- und Gefahrenquelle markiert wurden. Gleichwohl bemühte sich die Polizei auch um vertrauensbildende Maßnahmen und suchte bewusst die Nähe zu Bürger*innen mit Migrationsgeschichte.

Ein dritter Aspekt betriff die Deutungsfigur einer spezifischen Eigentümlichkeit der Kriminalität von „Fremden“ oder Minderheiten, wie sie im Begriff der „Ausländer-Kriminalität“ zum Ausdruck kommt, in dem sich eine komplexe Gemengelage gesellschaftlicher, politischer und polizeilicher Differenzzuschreibungen manifestiert. „Ausländer“ wurden in dieser Debatte also in gleich dreierlei Hinsicht zum Gegenstand sicherheitspolitischer Zuschreibungen: als (zukünftige) Akteure von Sicherheitsproduktion, als Adressaten einer spezifischen polizeilichen Sicherheitskommunikation und als potentielles Sicherheitsrisiko (vgl. auch Hunold et al. 2010, S. 11). Die Frage nach dem langfristigen Einfluss von ethnischer Diversität auf einen Wandel innerhalb der Organisation Polizei und auch im Verhältnis zum polizeilichen Publikum bleibt aus zeithistorischer Per- 
spektive ein lohnenswertes Unterfangen. Eine Integration von Menschen mit Migrationsgeschichte in die Cop Culture scheint indes auch heute noch einherzugehen mit einem hohen Assimilierungsdruck: „Migranten können zwar formal in die Polizei hinein gelangen, aber nur insoweit sie das traditionelle Selbstverständnis der Organisation nicht in Frage stellen bzw. irritieren" (Behr 2016, S. 20).

\section{Polizei und Sexualität: Annäherungen an die homosexuelle Community und queere Cops}

Die Geschichte Berlins ist eng verflochten mit der Geschichte der deutschen Homosexuellenbewegung im Spannungsfeld von Repression und Verfolgung, Emanzipation und Anerkennung. Seit Beginn des 20. Jahrhunderts und mit einer Blüte in den 1920er Jahren etablierte sich Berlin als „birthplace of a modern identity“ (Beachy 2014) als kosmopolitische schwule Hauptstadt Europas. Dies war auch deshalb möglich, weil die polizeilichen Strategien der Homosexuellenüberwachung hier weniger auf rigorose Repressionen als auf eine vergleichsweise „liberale“ Duldung homosexueller Räume setzte (vgl. Beachy 2014; Dobler 2008 u. 2020). Gleichwohl war Homosexualität in den 1920er Jahren ein Straftatbestand, und schwule Begegnungsorte wurden vielfach polizeilich überwacht. Die relative Freiheit zwischen „Duldungspolitik und Verbrechensbekämpfung“ (Dobler 2008) endete abrupt mit der Machtübernahme der Nationalsozialisten 1933, der Verschärfung des Strafgesetzparagraphen 175 und der Intensivierung der Homosexuellenverfolgung in den Folgejahren (dazu etwa Jellonnek 1990; Jellonnek/Lautmann 2002; Zinn 2018). ${ }^{10}$ Auch nach Ende des NS-Regimes lässt sich eine Kontinuität von polizeilicher Verfolgung und strafrechtlicher Diskriminierung beobachten, die erst mit der Großen Strafrechtsreform 1969 und der damit einhergehenden Entkriminalisierung einvernehmlicher homosexueller Beziehungen unter Männern abgemildert wurde. Verfolgungs- und Benachteiligungserfahrungen gehörten für die Betroffenen nach wie vor zum Alltag, wenngleich die Straffreiheit von Homosexualität unter erwachsenen Männern nunmehr einen

10 Neben der Verfolgung von homosexuellen Männern bzw. mann-männlicher Sexualität wird diskutiert, ob auch Lesben oder weitere queere Menschen explizit als aktiv von den Nationalsozialisten verfolgte (Opfer-)Gruppe gelten können (vgl. Dobler 2012, S. 61; Hájková 2018, S. 44f.; Zinn 2018). 
neuerlichen Schub für homosexuelle Emanzipationsbestrebungen bedeutete. Paragraph 175 wurde dennoch erst 1994 endgültig abgeschafft.

Diese kurze Chronologie verweist bereits auf das historisch hochgradig belastete Verhältnis von Homosexuellen und Polizei im Nachkriegsdeutschland. Von homosexuellen Menschen wurde die Polizei (verständlicherweise) lange Jahre als staatliche Verfolgungs- und Repressionsinstanz wahrgenommen. Dies führte auch dazu, dass Homosexuelle sich bei homophoben bzw. schwulen- und lesbenfeindlich motivierten ${ }^{11}$ Straftaten selten an Polizist*innen wandten. Homosexualität spielte (und spielt) auch innerhalb der Polizei eine Rolle, denn es kann davon ausgegangen werden, dass auch vor der Entkriminalisierung Homosexuelle in der Polizei tätig waren, die ihre sexuelle Orientierung nicht preisgaben. Erst selbstbewusste Forderungen der Homosexuellenbewegung und eine wachsende Zahl an Outings homosexueller Polizeibeamt"innen seit Beginn der 1990er Jahre veränderten den polizeilichen Umgang mit der Differenzkategorie sexuality (vgl. Dobler 2003).

Ende der 1980er und zu Beginn der 1990er Jahre erhielten insbesondere anti-schwule Gewalttaten und brutale Morde einige mediale Aufmerksamkeit. Das hing sicherlich auch damit zusammen, dass einige Betroffene und Aktivist"innen in der Schwulenbewegung diese Vorfälle nun bundesweit ins Licht der Öffentlichkeit rückten (vgl. Dobler 1996, S. 108f.). Antischwule Gewalt äußerte sich vor allem in teils brutalen Überfällen auf Homosexuelle an bekannten Treffpunkten, die von Neonazi-Gruppierungen, anderen Jugendbanden, aber auch homophoben Einzeltäter*innen, sogenannten „Schwulentickern“, verübt wurden (vgl. Kotte 1989; Der Spiegel 1992). An die Polizei hatte sich die überwiegende Mehrheit der Opfer solcher Gewaltdelikte zuvor kaum gewandt, vielmehr war die Beziehung zu den Ordnungshüter*innen geprägt von ausgesprochenem Misstrauen, waren doch, wie es die taz in einem Artikel aus dem Jahr 1989 beschreibt, „Klappenrazzien, Observationen mit Einwegspiegeln und polizeiliche Schwulenhatz in Parks aus den 70er Jahren den Schwulen noch in unguter Erinnerung" (Kotte 1989). Auch eine distanzierte polizeiliche Haltung gegenüber der schwulen Szene trug dazu bei, dass der Gewalt vorerst kein Einhalt geboten und die wenigen Anzeigen der Betroffenen nicht ernst genommen wurden. Ausschlaggebend dafür war ein ausgeprägtes polizeiliches Desinteresse gepaart mit Unwissenheit und Fehlannahmen in Bezug auf Charakteristika und Gepflogenheiten der Szene (vgl. Dobler 2003,

11 Auch Angehörige der LGBTQI*-Community wurden und werden in intersektionalen Bezügen häufig Opfer von Gewalttaten. 
S. 76-77). Das von sicherheitsstaatlicher Diskriminierung und Desinteresse sowie gegenseitigem Misstrauen geprägte Verhältnis wandelte sich erst in der ersten Hälfte der 1990er Jahre zaghaft - und das war vor allem darauf zurückzuführen, dass von einigen homosexuellen Aktivist"innen, neben Initiativen der Selbsthilfe (z. B. Überfalltelefone), nun eine Kooperation mit der Polizei regelrecht eingefordert wurde.

Im wiedervereinigten Berlin führte diese Entwicklung zur Berufung des bundesweit ersten „Schwulenbeauftragten“ in einer Polizeibehörde in Person von Heinz Uth, der in seiner Funktion als Kommissariatsleiter „Raub“ bei der Kriminalpolizei an der Aufklärung anti-schwuler Bandenkriminalität beteiligt war. Ihm war die mangelnde Anzeigenbereitschaft vieler Opfer homophober Gewalttaten aufgefallen, die fortan durch Vertrauenswerbung von Seiten der Polizei intensiviert werden sollte. Nach einer brutalen Razzia in einem bekannten Stricher-Lokal 1992, über die Uth trotz seiner Funktion nicht informiert worden war, setzte er weitergehende Kompetenzen und Aufgaben für sein Amt durch. Diese bestanden in erster Linie darin, einen direkten Kontakt zur homosexuellen Szene Berlins zu etablieren und präventiv über Hilfsmöglichkeiten bei anti-schwuler Gewalt aufzuklären, etwa durch eine enge Zusammenarbeit mit dem Berliner Überfalltelefon oder Inseraten in Szene-Magazinen. Zu diesem Zweck setzte die Polizei auch ein neuartiges Infomobil ein, das an bekannten Treffpunkten für Homosexuelle in Berlin für mehr Vertrauen in die Polizei warb. Hier engagierten sich auch offen homosexuell lebende Polizeibeamt*innen, für die Uth als vertrauensvoller Ansprechpartner galt (vgl. Bieniek 1993). Auf Uths Initiative ging auch 1993 das erste lesbisch-schwule Motzstraßenfests in Schöneberg zurück, das seither als ein wichtiges Zeichen gegen homophobe Gewalt und für stärkere Akzeptanz wie Solidarität mit der queeren Szene gilt. In der Gegenwart richtet sich die Berliner Polizei mit eigenen Ansprechpersonen neben Lesben und Schwulen auch an Bisexuelle sowie trans- und intergeschlechtliche Menschen. 1994 konstituierte sich, nur kurze Zeit nach der Abschaffung des Paragraph 175, der Verband lesbischer und schwuler Polizeibediensteter, die ihren „Anspruch auf Gleichbehandlung und Akzeptanz" nunmehr auch innerhalb der Organisation vertraten und dort mittlerweile überwiegend anerkannt sind (Terweide 2004).

Mit Blick auf die Differenzkategorie sexuality lässt sich im Verhältnis von Polizei und queerer Szene mit guten Gründen von einer gegenseitigen Annäherung und Akzeptanz oder gar einem langwierigen Normalisierungsprozess sprechen. Gleichwohl bleibt die Frage bestehen, wie sehr auch heute noch der polizeiliche Blick auf LGBTQI*-Personen von hartnäckig persistenten Stereotypen und Vorurteilen geprägt ist und welche Veränderungen die Annäherungsprozesse der 1990er Jahre innerhalb der 
nach wie vor überwiegend heteronormativ-maskulinen Polizeikultur angestoßen haben. Die 2005 bekanntgewordene Erfassung von Sexualpräferenzen polizeilich dokumentierter Personen (sog. „rosa Listen“) in einigen Polizeidatenbanken verweist auf die fortbestehende Verknüpfung von polizeilicher (Un-)Sicherheitsproduktion und sexueller Orientierung (vgl. taz 2005).

\section{Resümee: Sicherheit, Differenz und Polizeigeschichte}

Dieser Beitrag hat den Versuch unternommen, anhand der Kategorien gender, race/ethnicity und sexuality den oftmals widersprüchlichen Zusammenhang und die vielfältigen Berührungspunkte von Differenz und Sicherheit am historischen Beispiel der (West-)Berliner Polizei und ihrem häufig konflikthaften Verhältnis zur Stadtbevölkerung im Spannungsfeld von Mehrheits- und Minderheitsgesellschaft sowie von Sicherheitsproduktion und Bedrohungsinstanz deutlich zu machen. Innerhalb der West-Berliner Polizei wurde, wie auch in anderen Polizeien in West-Deutschland, eine maskulin-zupackende, soldatische „Krieger-Männlichkeit“ kultiviert, die auch das Verhältnis zu jugendkulturellem und studentischem Protest, mithin den Blick auf gesellschaftliche Devianz und entsprechende Einsatztaktiken prägte. Diese Vorstellung von Polizeiarbeit wandelte sich erst langsam in einer Zeit der Reformen ab den späten 1960er Jahren. Eine erste Phase der Integration von Frauen in die Berliner Schutzpolizei in Form der WSP war von dem aus der Weimarer Republik tradierten Bild weiblich-fürsorgender Polizeiarbeit geprägt, das die hegemonial-männliche Cop Culture jener Jahre kaum beeinflusste. Auch nach der Öffnung der Schutzpolizei Ende der 1970er Jahre blieb zunächst die Vorstellung von „weiblicher Eigenart" erhalten. Auch wenn sich das inzwischen deutlich gewandelt hat, bleibt die Frage nach einer Persistenz hegemonialer Männlichkeitsvorstellungen in der gegenwärtigen Polizeikultur bestehen. Im Verhältnis zwischen West-Berliner Polizei und „Ausländern“ wurde zunächst intensiv verhandelt, wer das staatliche Gewaltmonopol vertreten solle und ob Immigrant"innen dafür in Frage kämen. Gleichermaßen war die Polizeipraxis jener Jahre von diskriminierenden Differenzkonstruktionen geprägt, die wiederum mit Vorstellungen einer spezifischen „Ausländer-Kriminalität“ einhergingen und dergestalt versicherheitlicht wurden. Die Untersuchung der Differenzkategorien race bzw. ethnicity kann also dabei helfen, das Verhältnis von Polizei und Gesellschaft um die Perspektive bislang vernachlässigter (und potentiell strukturell diskriminierter) Akteursgruppen zu erweitern. Das von gegenseitigem Misstrauen und historischer Vorbelas- 
tung geprägte Verhältnis von Homosexuellen und (West-)Berliner Polizei entschärfte sich erst Anfang der 1990er Jahre. Im Vergleich zu den 1950er und 1960er Jahren hat sich die Polizei zu einer deutlich bürgernäheren, diversifizierten und rechtsstaatlich orientierten Institution des staatlichen Gewaltmonopols entwickelt. Dies sollte jedoch nicht als eine lineare Fortschrittsgeschichte (fehl-)gedeutet werden, läuft doch die „Differenzmaschine“ Polizei im „Alltag des Gewaltmonopols“ (Behr 2008) unablässig weiter und produziert weitere (Un-)Sicherheiten.

Die Differenzkategorien gender, race/ethnicity und sexuality sind in diesem Aufsatz vor allem aus forschungspragmatischen und darstellerischen Gründen etwas unverbunden nebeneinandergestellt worden. Gleichwohl lassen sich viele intersektionale Bezüge und (Quer-)Verbindungen aufzeigen, die eine Beschäftigung mit Polizei und Differenz aus historischer Perspektive bereichern können. Dabei ließe sich erstens an Überschneidungen zwischen unterschiedlichen Differenzkategorien und -zuschreibungen denken, etwa die Erfahrungen von Frauen mit Migrationsgeschichte im Umgang mit der Polizei oder die spezifischen Erfahrungen von lesbischen Polizeibeamtinnen (vgl. Marach 1996). Zweitens könnten weitere Gruppen als die in diesem Text genannten in den Mittelpunkt gerückt werden. Drittens lässt sich neben Unterschieden und Grenzziehungen zwischen den vielen „Anderen“ in Polizei und Gesellschaft sicher noch stärker den Gemeinsamkeiten nachspüren. Dies betriff insbesondere die Frage, mit welchen zum Teil ähnlichen Anpassungsmechanismen zwischen Integrationsanspruch und Assimilierungszwang die Polizei unterschiedliche gesellschaftliche Gruppen in ihre Organisationskultur aufnahm - und welche nicht.

Der hier vorgenommene historische Überblick hat gezeigt, welchen Mehrwert die Analysekategorie Differenz in unterschiedlichen Ausprägungen für eine Polizeigeschichte der Bundesrepublik generieren kann, die sich explizit auch als Gesellschaftsgeschichte versteht (vgl. Mecking 2020). Auf ein eindimensionales Narrativ lässt sich diese Geschichte kaum reduzieren: Eine derart differenzierende Polizeigeschichte der Bundesrepublik wird sich wohl nur entlang der durchaus widersprüchlichen Prozesse von langwierigen, nicht linear verlaufenden Emanzipationsprozessen unterschiedlicher gesellschaftlicher Gruppen und Minderheiten in und außerhalb der Polizei sowie einer ungemein persistenten hegemonialen Cop Culture erzählen lassen. 


\section{Literatur}

Acker, Joan (1990): Hierarchies, Jobs, Bodies. A Theory of Gendered Organizations. In: Gender \& Society 4, H. 2, S. 139-158.

Alexopoulou, Maria (2019): 'Ausländer' - A Racialized Concept? 'Race' as an Analytical Concept in Contemporary German Immigration History. In: Arghavan, Mahmoud/Hirschfelder, Nicole/Kopp, Luvena/Motyl, Katharina (Hrsg.): Who Can Speak and Who is Heard/Hurt? Facing Problems of Race, Racism, and Ethnic Diversity in the Humanities in Germany. Bielefeld: transcript, S. 45-67.

Beachy, Robert (2014): Gay Berlin. Birthplace of a Modern Identity. New York: Alfred A. Knopf.

Behr, Rafael (2008): Cop Culture - Der Alltag des Gewaltmonopols. Männlichkeit, Handlungsmuster und Kultur in der Polizei. Wiesbaden: Springer VS.

Behr, Rafael (2016): Diversität und Polizei. Eine polizeiwissenschaftliche Perspektive. In: Gekova, Petia/Ringeisen, Tobias (Hrsg.): Handbuch Diversity Kompetenz. Band 1: Perspektiven und Anwendungsfelder. Wiesbaden: Springer VS, S. 557-578.

Behr, Rafael (2017): Maskulinität in der Polizei: Was Cop Culture mit Männlichkeit zu tun hat. Ein Essay. In: juridikum 4/2017, S. 541-551.

Behr, Rafael (2018): „Die Polizei muss ... an Robustheit deutlich zulegen“: Zur Renaissance aggressiver Maskulinität in der Polizei. In: Loick, Daniel (Hrsg.): Kritik der Polizei. Frankfurt a. M./New York: Campus, S. 165-178.

Beil, Jens (1968): „Brigade des Charmes“ auch in Berlin? In: Welt am Sonntag vom 17.11.1968.

Bentheim, Ursula von (1980): Mit Charme und kesser Mütze regeln weiße Mäuschen den Verkehr, In: Berliner Morgenpost, 26.09.1980.

Bieniek, Jürgen (1993): Schwule Polizei - dein Freund und Helfer? In: taz vom 02.09.1993.

Blankenburg, Erhard/Feest, Johannes (1972): Die Definitionsmacht der Polizei. Strategien der Strafverfolgung und soziale Selektion. Düsseldorf: Bertelsmann Universitätsverlag.

Blum, Bettina (2012): Polizistinnen im geteilten Deutschland. Geschlechterdifferenz im staatlichen Gewaltmonopol vom Kriegsende bis in die siebziger Jahre. Essen: Klartext Verlag.

Blum, Bettina (2015): Die Geschichte der Frauen in der Polizei im 20. Jahrhundert. In: Oranienburger Schriften 1/2015, S. 120-129.

Blumenthal, Dirk/Fritz, Detlef (1984): Schnüffelei im Schlafzimmer. In: Stern vom 23.08.1984.

Borgmann, Malte (2019): Von der Anwerbung zur Anerkennung. Migration, Politik und Teilhabe in West-Berlin 1970-1984. In: Hochmuth, Hanno/Nolte, Paul (Hrsg.): Stadtgeschichte als Zeitgeschichte. Berlin im 20. Jahrhundert. Göttingen: Wallstein, S. 271-297. 
Brusten, Manfred/Feest, Johannes/Lautmann, Rüdiger (Hrsg.) (1975): Die Polizei - eine Institution öffentlicher Gewalt. Analysen, Kritik, empirische Daten. Neuwied u.a.: Luchterhand.

Buzan, Barry/Waever, Ole/de Wilde, Jaap (1997): Security. A New Framework for Analysis. Boulder, CO: Lynne Rienner.

Conze, Eckart (2005): Sicherheit als Kultur. Überlegungen zu einer „modernen Politikgeschichte" der Bundesrepublik Deutschland. In: Vierteljahrshefte für Zeitgeschichte 53, H. 3, S. 357-380.

Dams, Carsten (2008): Die Polizei in Deutschland 1945-1989. In: Aus Politik und Zeitgeschichte 48/2008, S. 9-14.

Der Abend (1968): ,Wespen“ mit schlanker Taille. In: Der Abend vom 11.10.1968.

Der Spiegel (1972): „Hallo, Fräulein“. In: Der Spiegel, 35/1972.

Der Spiegel (1974): Stopp für Türken. In: Der Spiegel 45/1974.

Der Spiegel (1979a): „Wir müssen Signale geben“. In: Der Spiegel 37/1979.

Der Spiegel (1979b): Gepriesene Gleichheit (Leserbriefe). In: Der Spiegel 40/1979.

Der Spiegel (1979c): Viel Glück (Leserbriefe). In: Der Spiegel 42/1979.

Der Spiegel (1992): „Jeder hat mal reingetreten“. In: Der Spiegel 12/1992.

Der Tagesspiegel (1982a): Wo Schlepper und Schleuser verkehren. In: Der Tagesspiegel vom 03.09.1982.

Der Tagesspiegel (1982b): „Um Anerkennung hat die Polizistin kämpfen müssen“. In: Der Tagesspiegel vom 21.11.1982.

Diederichs, Otto (1986): Die AGA - Sonderfahnder gegen Ausländer. In: Bürgerrechte \& Polizei/CILIP 24 (2/1986), S. 46-51.

Diederichs, Otto (1993): Die ,Arbeitsgruppe Ausländer' der Berliner Polizei - eine Sondereinheit mit Doppelfunktion. In: Bürgerrechte \& Polizei/CILIP 45 (2/1993), S. 46-49.

Diewald-Kerkmann, Gisela (2009): Frauen, Terrorismus und Justiz. Prozesse gegen weibliche Mitglieder der RAF und der Bewegung 2. Juni. Düsseldorf: Droste Verlag.

Dobler, Jens (1996): Antischwule Gewalt und Polizei. In: Ders. (Hrsg.): Schwule, Lesben, Polizei. Vom Zwangsverhältnis zur Zweck-Ehe? Berlin: Verlag rosa Winkel, S. 107-113.

Dobler, Jens (2003): Antischwule Gewalt: Hintergründe und Gegenperspektiven. In: Koher, Frauke/Pühl, Katharina (Hrsg.): Gewalt und Geschlecht. Konstruktionen, Positionen, Praxen. Wiesbaden: Springer VS 2003, S. 67-81.

Dobler, Jens (2008): Zwischen Duldungspolitik und Verbrechensbekämpfung. Homosexuellenverfolgung durch die Berliner Polizei von 1848 bis 1933. Frankfurt a. M.: Verlag für Polizeiwissenschaft.

Dobler, Jens (2012): Unzucht und Kuppelei. Lesbenverfolgung im Nationalsozialismus. In: Eschebach, Insa (Hrsg): Homophobie und Devianz. Weibliche und männliche Homosexualität im Nationalsozialismus. Berlin: Metropol Verlag, S. 53-62. 
Dobler, Jens (2020): Polizei und Homosexuelle in der Weimarer Republik. Zur Konstruktion des Sündenbabels. Berlin: Metropol Verlag.

Dudek, Sonja M. (2009): Diversity in Uniform? Geschlecht und Migrationshintergrund in der Berliner Schutzpolizei. Wiesbaden: Springer VS.

Fürmetz, Gerhard/Reinke, Herbert/Weinhauer, Klaus (Hrsg.) (2001): Nachkriegspolizei. Sicherheit und Ordnung in Ost- und Westdeutschland 1945-1969. Hamburg: Ergebnisse Verlag.

Fürmetz, Gerhard (Hrsg.) (2006): „Schwabinger Krawalle“. Protest, Polizei und Öffentlichkeit zu Beginn der 60er Jahre. Essen: Klartext Verlag.

Gaserow, Vera (1985): Mit Kennwort „Advocat“ auf der Suche nach Dunkelhäutigen. In: taz vom 25.04.1985.

Götting, Dirk (2009): Die ,Weibliche Kriminalpolizei‘; ein republikanisches Reformprojekt zwischen Krise und Neuorientierung im Nationalsozialismus. In: Schulte, Wolfgang (Hrsg.): Die Polizei im NS-Staat. Beiträge eines internationalen Symposiums an der Deutschen Hochschule der Polizei in Münster, Frankfurt a. M.: Verlag für Polizeiwissenschaft, S. 481-510.

Götting, Dirk (2010): Das Aufbegehren der bürgerlichen Frauenbewegung gegen die Sittenpolizei des Kaiserreichs und der erste Versuch weiblicher Polizeiarbeit in Deutschland (1875-1914). Frankfurt a. M.: Verlag für Polizeiwissenschaft.

Grun, Brigitte (1982): „14. Landesdelegiertentag“ - „Frauen bei der Polizei“, nicht neu, doch wir Schutzpolizistinnen wurden wieder neugeboren. In: Polizeispiegel (Berlin) 7/8/1982, S. 71.

Haibach, Hans (1979): Jeder fünfte Kreuzberger ist ein Türke. In: Frankfurter Allgemeine Zeitung vom 27.10.1979.

Hájková, Anna (2018): Queere Geschichte und der Holocaust. In: Aus Politik und Zeitgeschichte 38-39/2018, S. 42-47.

Hansen, Lene (2000): The Little Mermaid's Silent Security Dilemma and the Absence of Gender in the Copenhagen School. In: Millennium - Journal of International Studies 29, H. 2, S. 285-306.

Herbert, Ulrich (2001): Geschichte der Ausländerpolitik in Deutschland. Saisonarbeiter, Zwangsarbeiter, Gastarbeiter, Flüchtlinge. München: Beck.

Hunold, Daniela (2010): Vom Promille zum Prozent. Der Bestand an Polizeibeamten mit migrantischem Hintergrund in den Bundesländern. In: Dies./Klimke, Daniela/Behr, Rafael/Lautmann, Rüdiger (Hrsg.): Fremde als Ordnungshüter? Die Polizei in der Zuwanderungsgesellschaft Deutschland. Wiesbaden: Springer VS, S. 137-143.

Hunold, Daniela/Klimke, Daniela/Behr, Rafael/Lautmann, Rüdiger (2010): Einleitung: Die Integration von Migranten in die Polizeiorganisation. In: Dies. (Hrsg.): Fremde als Ordnungshüter? Die Polizei in der Zuwanderungsgesellschaft Deutschland. Wiesbaden: Springer VS, S. 9-25.

Hunold, Daniela/Wagner, Maren (2020): Rassismus und Polizei. Zum Stand der Forschung. In: Aus Politik und Zeitgeschichte 42-44/2020, S. 27-32.

Jellonnek, Burkhard (1990): Homosexuelle unter dem Hakenkreuz. Die Verfolgung von Homosexuellen im Dritten Reich. Paderborn: Schöningh. 
Jellonnek, Burkhard/Lautmann, Rüdiger (2002) (Hrsg.): Nationalsozialistischer Terror gegen Homosexuelle. Verdrängt und ungesühnt. Paderborn: Schöningh.

Kleinknecht, Thomas/Sturm, Michael (2004): „Demonstrationen sind punktuelle Plebiszite“. Polizeireform und gesellschaftliche Demokratisierung von den Sechziger- zu den Achtzigerjahren. In: Archiv für Sozialgeschichte 44, S. 181-218.

Könne, Christian (2018): Homosexuelle und die Bundesrepublik Deutschland. Gleichberechtigte Mitmenschen? www.bpb.de/geschichte/zeitgeschichte/deut schlandarchiv/275113/homosexuelle-und-die-bundesrepublik-deutschland, 07.04.2021.

Kotte, Hans-Hermann (1989): Schwule ticken im Park - quasilegale Gewalt im Dunkeln. In: taz vom 15.06.1989.

Lindenberger, Thomas (2003): Volkspolizei. Herrschaftspraxis und öffentliche Ordnung im SED-Staat 1952-1968. Köln/ Weimar/Wien: Böhlau.

Hübner, Klaus (1952): Katze aus dem Sack: Jetzt weht ein anderer Wind... In: Deutsche Polizei 7/1952, S. 1-3.

Loick, Daniel (2018): Was ist Polizeikritik? In: Ders. (Hrsg.): Kritik der Polizei. Frankfurt a. M./New York: Campus, S. 9-35.

Luft, Stefan (2014): Die Anwerbung türkischer Arbeitnehmer und ihre Folgen. www.bpb.de/internationales/europa/tuerkei/184981/gastarbeit, 07.04.2021.

Marach, Gabriele (1996): Von einer Lesbe, die auszog, Polizistin zu werden. In: Dobler, Jens (Hrsg.): Schwule, Lesben, Polizei. Vom Zwangsverhältnis zur ZweckEhe? Berlin: Verlag rosa Winkel, S. 155-158.

Mecking, Sabine (2020): Mehr als Knüppel und Knöllchen: Polizeigeschichte als Gesellschaftsgeschichte. In: Dies. (Hrsg.): Polizei und Protest in der Bundesrepublik Deutschland. Wiesbaden: Springer VS, S. 1-25.

Münch, Ursula (1993): Asylpolitik in der Bundesrepublik Deutschland. Entwicklung und Alternativen. 2., aktual. Aufl., Opladen: Leske + Budrich.

Neurath, Wolf-Rüdiger (1980): Der Schutzpolizistin stehen alle polizeilichen Tätigkeiten offen. „Karate-Emmas“ sind jedoch nicht gefragt. In: Stuttgarter Nachrichten vom 11.07.1980.

Nienhaus, Ursula (1999): „Nicht für eine Führungsposition geeignet“. Josephine Erkens und die Anfänge weiblicher Polizei in Deutschland 1923-1933. Münster: Westfälisches Dampfboot.

Nienhaus, Ursula (2001): „Für strenge Dienstzucht ungeeignete Objekte ..."Weibliche Polizei in Berlin 1945-1952. In: Fürmetz, Gerhard/Reinke, Herbert/Weinhauer, Klaus (Hrsg.): Nachkriegspolizei. Sicherheit und Ordnung in Ost- und Westdeutschland 1945-1969. Hamburg: Ergebnisse Verlag, S. 129-153.

Pfennig, Gerhard (1978): Die Frau in der Polizei. In: Die Polizei 69, H. 2, S. 40-43.

Polizeischau (1975): Ausländer-Arbeitsgruppen haben sich bewährt. In: Polizeischau. Zeitschrift für die Berliner Polizei 5/1975, S. 4-6.

Polizeischau (1980): Frauen bei der Polizei kamen gut an. In: Polizeischau. Zeitschrift für die Berliner Polizei 10/1980, S. 4. 
Rommelspacher, Birgit (2009): Was ist eigentlich Rassismus? In: Mecheril, Paul/ Melter, Claus (Hrsg): Rassismuskritik. Band I: Rassismustheorie und -forschung. Schwalbach/Taunus: Wochenschau Verlag, S. 25-38.

Schmeer, Marcel (2020): Soziologen, Straßenkämpfer, Psychobullen. Die West-Berliner Polizei als umstrittene Organisation. In: Böick, Marcus/Schmeer, Marcel (Hrsg.): Im Kreuzfeuer der Kritik. Umstrittene Organisationen im 20. Jahrhundert. Frankfurt a. M./New York: Campus, S. 285-321.

Statistisches Landesamt Berlin (Hrsg.) (1968): Statistisches Jahrbuch Berlin 1968. Berlin: Kulturbuch-Verlag.

Statistisches Landesamt Berlin (Hrsg.) (1976): Statistisches Jahrbuch Berlin 1976. Berlin: Kulturbuch-Verlag.

Statistisches Landesamt Berlin (Hrsg.) (1981): Statistisches Jahrbuch Berlin 1981. Berlin: Kulturbuch-Verlag.

Steinborn, Norbert/Krüger, Hilmar (1993): Die Berliner Polizei 1945-1992. Von der Militärreserve im Kalten Krieg auf dem Weg zur bürgernahen Polizei? Berlin: Berlin-Verlag Spitz.

Stokes, Lauren (2020): Racial Profiling im Kalten Krieg. Honeckers fliegender Teppich. In: Die Zeit, 30.06.2020. www.zeit.de/2020/32/racial-profiling-ddr-eric h-honecker-fluechtlinge-ostberllin-westberlin, 07.04.2021.

Süddeutsche Zeitung (1978): Westberliner Polizei kontrolliert erstmals in S-Bahnhöfen. In: Süddeutsche Zeitung vom 12.09.1978.

Süddeutsche Zeitung (1980): Charme soll für Ordnung sorgen. In: Süddeutsche Zeitung vom 30.09.1980.

taz (2005): NRW löscht „rosa Listen“. In: taz vom 10.08.2005.

Terweide, Annette (2004): Lesben und Schwule in der Polizei. Anspruch auf Gleichbehandlung und Akzeptanz. Haan: Wölfer Druck.

Tielemann, Kea (1993): Frauen in der Schutzpolizei. In: Bürgerrechte \& Polizei/ CILIP 46 (3/1993), S. 18-22.

Ulrich, Peter (1979): Ein Zeichen der Integration. In: Polizeischau. Zeitschrift für die Berliner Polizei 7/1979, S. 2.

Weinhauer, Klaus (2001): Innere Unruhe. Studentenproteste und die Krise der westdeutschen Schutzpolizei in den sechziger Jahren. In: Fürmetz, Gerhard/ Reinke, Herbert/Ders. (Hrsg.): Nachkriegspolizei. Sicherheit und Ordnung in Ost- und Westdeutschland 1945-1969. Hamburg: Ergebnisse Verlag, S. 303-325.

Weinhauer, Klaus (2003): Schutzpolizei in der Bundesrepublik. Zwischen Bürgerkrieg und Innerer Sicherheit: Die turbulenten sechziger Jahre. Paderborn: Schöningh.

Weinhauer, Klaus (2008): Zwischen Tradition und Umbruch. Schutzpolizei in den 1950er bis 1970er Jahren (Personal, Ausbildung, Revierdienst, Großeinsätze). In: Leßmann-Faust, Peter (Hrsg.): Polizei und Politische Bildung. Wiesbaden: Springer VS, S. 21-43. 
Weinhauer, Klaus (2020): Zwischen organisatorischen Wandlungen und kulturellen Kontinuitäten. Polizei, Jugendprotest und Demonstrationen in den 1960er bis 1980er Jahren. In: Mecking, Sabine (Hrsg.): Polizei und Protest in der Bundesrepublik Deutschland. Wiesbaden: Springer VS, S. 165-184.

Weis, Otto Jörg (1979): Wachtmeister Nihat Dogru geht jetzt Streife in Kreuzberg. In: Hannoversche Allgemeine vom 27.09.1979.

Weis, Otto Jörg (1980): Auch Polizistinnen müssen notfalls schießen. In: Frankfurter Rundschau vom 22.07.1980.

West, Candace/Fenstermaker, Sarah (1995): Doing Difference. In: Gender and Society 9 , H. 1, S. 8-37.

Wilz, Sylvia (2012): Die Polizei als Organisation. In: Apelt, Maja/Tacke, Veronika (Hrsg.): Handbuch Organisationstypen. Wiesbaden: Springer VS, S. 113-131.

Wüller, Heike (2010): Presseschau: Die Haltung der polizeilichen Berufsvertretungen zur Diversität in den eigenen Reihen. In: Hunold, Daniela/Klimke, Daniela/ Behr, Rafael/Lautmann, Rüdiger (Hrsg.): Fremde als Ordnungshüter? Die Polizei in der Zuwanderungsgesellschaft Deutschland. Wiesbaden: Springer VS, S. $157-176$.

Zeit Online (2020): Zehntausende Menschen protestieren deutschlandweit gegen Rassismus. www.zeit.de/gesellschaft/zeitgeschehen/2020-06/demonstration-anti-r assismus-polizeigewalt-deutschland-protest-black-lives-matter, 07.04.2021.

Zinn, Alexander (2018): Aus dem Volkskörper entfernt? Homosexuelle Männer im Nationalsozialismus. Frankfurt a. M./New York. 